\title{
X-Ray Interferometry with Microelectronvolt Resolution
}

\author{
Yu.V. Shvyd'ko, * M. Lerche, H.-C. Wille, E. Gerdau, M. Lucht, and H. D. Rüter \\ Institut für Experimentalphysik, Universität Hamburg, D-22761 Germany \\ E. E. Alp and R. Khachatryan \\ Advanced Photon Source, Argonne National Laboratory, Argonne, Illinois 60439
}

(Received 5 August 2002; published 10 January 2003)

\begin{abstract}
We demonstrate an interferometer for hard $\mathrm{x}$ rays with two back-reflecting sapphire crystal mirrors - a prototype x-ray Fabry-Pérot interferometer. A finesse of 15 and $0.76 \mu \mathrm{eV}$ broad FabryPérot transmission resonances are measured by the time response of the interferometer. Interference patterns are observed directly in spectral dependences of reflectivity.
\end{abstract}

Microscopes, spectrometers, interferometers, and other high-resolution optical devices are indispensable in natural sciences. Invented by Fabry and Pérot [1] the interferometers known under their names have been used for more than a century to explore the visible spectral region of the electromagnetic radiation. They are used for highprecision wavelength measurements in atomic spectroscopy, in astrophysics, and in many other physical as well as life sciences [2,3]. They are used as interference filters and resonators in laser physics. High-resolution instruments of this kind would be extremely useful for research with $\mathrm{x}$ rays. X-ray Fabry-Pérot interferometers (or resonators) could be used as interference filters with micro-eV spectral resolution [4] for studying dynamics of solids, liquids, and macroscopic biological molecules. They could be used for phase-contrast imaging of nanometerscale objects. A combined optical-x-ray Fabry-Pérot interferometer could bridge the optical and $\mathrm{x}$-ray domains [5] by measuring directly the $\mathrm{x}$ ray to visible light wavelength ratios [6]. X-ray Fabry-Pérot interferometers present an exciting and challenging problem.

The main components of the simplest Fabry-Pérot interferometer are two highly reflecting low absorbing parallel mirrors. The system becomes transparent despite the high reflectivity of each mirror when the gap $d_{\mathrm{g}}$ between the mirrors is an integer multiple of half of the radiation wavelength as then the resonance condition for standing wave formation in the gap is fulfilled. The energy separation between two successive transmission resonances the free spectral range $E_{\mathrm{f}}=h c / 2 d_{\mathrm{g}}$ - is a constant independent of the incident photon energy. The spectral width of the transmission resonances $\Gamma=E_{\mathrm{f}} / F$ is smaller the higher the finesse $F=\pi \sqrt{R} /(1-R)$, or equivalently, the higher the mirror reflectivity $R$ [2]. With a reflectivity of $R=0.85(F=19.3)$ and a gap of $50 \mathrm{~mm}$, the width of the transmission resonances is in the sub-micro-eV range. Physically, such a small spectral width is due to interference of a large number $\simeq F$ of coherent waves arising from multiple reflections.
Beam divergence blurs the resonances and degrades the interferometer's performance. To avoid this, the back-reflection geometry is essential. Unlike the visible spectral region where back-reflection mirrors of high reflectivity are available, this is a problem for $\mathrm{x}$ rays.

Steyerl and Steinhauser [4] have pioneered the idea of a Fabry-Pérot-type interference filter for $\mathrm{x}$ rays proposing the replacement of the optical mirrors by Bragg backreflection from parallel-sided silicon single crystals. The theory of such devices was propounded by Caticha et al. $[10,11]$. A revised theory taking also into account possible imperfections of the x-ray Fabry-Pérot interferometers was given by Kohn et al. [12]; see also [5,13].

Liss et al. [14] have observed multiple reflections of $\mathrm{x}$ rays in backscattering from a system of two parallel silicon single crystals. The intensity of the reflected beams was, however, too low to be useful, because of the low reflectivity of silicon crystals in backscattering arising from parasitic Bragg reflections [15,16]. As distinct from silicon, sapphire $\left(\alpha-\mathrm{Al}_{2} \mathrm{O}_{3}\right)$ provides the required high reflectivity in backscattering $[5,15]$. Recently it was demonstrated that a system of two parallel $\alpha-\mathrm{Al}_{2} \mathrm{O}_{3}$ crystal mirrors can produce a large number of intense overlapping beams [5,17]. In this Letter we present first direct evidences of the interference of the waves reflected from two separate sapphire crystals in backscattering.

The results, which we are reporting here, were obtained at the undulator beam line ID3 at the synchrotron radiation facility APS (ANL, Argonne). Many years of preliminary studies were carried out at the wiggler beam line BW4 at HASYLAB (DESY, Hamburg).

The (0 0030$)$ Bragg reflection in $\alpha-\mathrm{Al}_{2} \mathrm{O}_{3}$ single crystals was used to reflect $14.315 \mathrm{keV}$ x rays backwards. Crystals were grown by the heat-exchange method [18]. Dislocation-free samples were selected by white beam $\mathrm{x}$-ray topography [19]. According to dynamical theory calculations, a peak reflectivity of $R=0.85$ can be achieved by using a $d \simeq 60 \mu \mathrm{m}$ thick $\alpha-\mathrm{Al}_{2} \mathrm{O}_{3}$ crystal 
plate [5,13]. The width of the reflectivity region is $17.2 \mathrm{meV}$. The energy dependence of reflectivity and transmissivity in exact backscattering for two $\alpha-\mathrm{Al}_{2} \mathrm{O}_{3}$ crystal plates picked out for the interferometer mirrors is shown in Fig. 1. The measurements were performed with $\mathrm{x}$ rays monochromatized to a bandwidth of $2 \mathrm{meV}$ [20] and directed normal to the mirrors with an accuracy of $5 \mu \mathrm{rad}$. Beam divergence was $15 \times 40 \mu \mathrm{rad}^{2}$.

The dependences for mirror 1 in Fig. 1(a) and mirror 2 in Fig. 1(b) look very similar. The peak reflectivities are almost the same: $R=0.84(2)$. Still, a slightly different period of oscillations on the wings can be observed. The oscillations are due to the interference of the waves reflected from the front and the rear crystal surfaces. The period is $E_{\mathrm{d}}=h c / 2 d[5,13]$, with $h$ as the Planck constant, and $c$ as the speed of light in vacuum. This simple relation allows one to directly ascertain the crystal thicknesses to be $d_{1}=63.0(1) \mu \mathrm{m}$ and $d_{2}=$ 58.6(2) $\mu \mathrm{m}$ for mirrors 1 and 2, respectively. The small difference $\delta d=d_{1}-d_{2}=4.4 \mu \mathrm{m}$ in the mirror thicknesses will become important in the experiment, which proves the functioning of the interferometer (Fig. 4). The comparison of the energy dependencies both measured and calculated by the dynamical theory of $\mathrm{x}$-ray diffraction shows very good agreement. The mismatch in the amplitudes of the oscillations is attributed to a small thickness inhomogeneity of the mirrors. Built of such mirrors, a Fabry-Pérot interferometer should have a finesse of $F=18(3)$.

Figure 2 shows simulations of the reflectivity and transmissivity of an x-ray Fabry-Pérot interferometer with such mirrors. The theory $[12,5,13]$ was applied. The mirrors in the interferometer are at a distance of $d_{\mathrm{g}} \simeq$ $50 \mathrm{~mm}$ chosen for reasons of the accessible time resolution, which is discussed below. The Fabry-Pérot resonan-

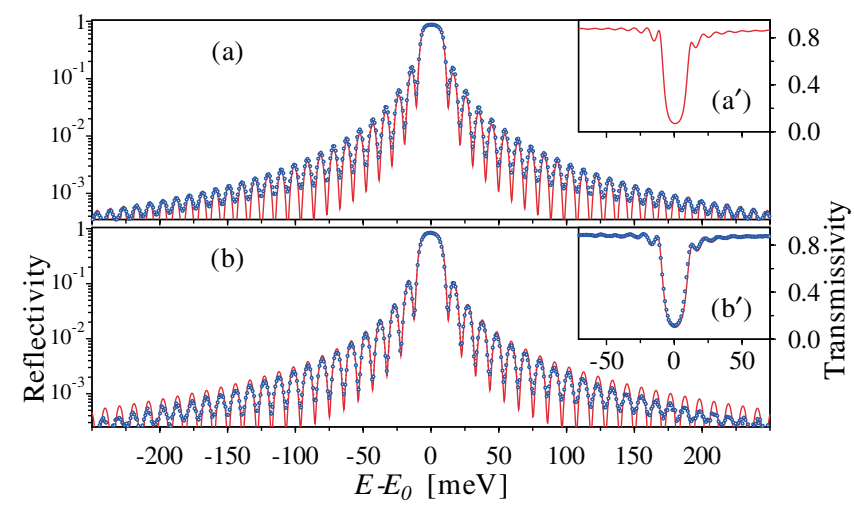

FIG. 1 (color). Energy dependence of the reflectivity (a),(b), and transmissivity $\left(\mathrm{a}^{\prime}\right),\left(\mathrm{b}^{\prime}\right)$, of $\mathrm{x}$ rays at normal incidence to the

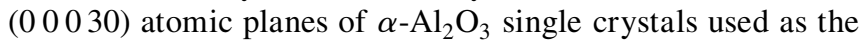
interferometer mirrors: (a), $\left(\mathrm{a}^{\prime}\right)$ : mirror $1, d_{1}=63.0(1) \mu \mathrm{m}$; (b), $\left(\mathrm{b}^{\prime}\right)$ : mirror $2, d_{2}=58.6(2) \mu \mathrm{m}$ thick. $E_{0}=14.315 \mathrm{keV}$. Red lines are theoretical fits by using the dynamical theory of $\mathrm{x}$-ray diffraction in exact backscattering. In the fit, the $2 \mathrm{meV}$ energy width of the incident radiation is taken into account. ces appearing due to the multiple-beam interference are $0.64 \mu \mathrm{eV}$ broad and spaced out $E_{\mathrm{f}}=12.4 \mu \mathrm{eV}$ apart. There exists no x-ray monochromator, which could be used to resolve such sharp resonances. However, since time and energy are complementary, the sharp energy resonances could be detected in the time response of the Fabry-Pérot interferometer. The time response to the excitation with a $\delta$-function-like radiation pulse should be a decaying exponential function with superimposed oscillations [5,13]. The decay constant is $\tau=\hbar / \Gamma$. The oscillations are a kind of quantum beating due to interference of the path amplitudes in scattering via neighboring resonances spaced out by $E_{\mathrm{f}}$ apart. The quantum beat period is $t_{\mathrm{f}}=2 \pi \hbar / E_{\mathrm{f}} \equiv 2 d_{\mathrm{g}} / c$. Thus, $t_{\mathrm{f}}$ is also equal to the round-trip circulation time in the x-ray cavity, and to the time lag between successive multiply-scattered beams. With the given finesse and the chosen gap one may expect $\tau=1.1 \mathrm{~ns}$, and $t_{\mathrm{f}}=333 \mathrm{ps}$ for such an interferometer. Both values can be measured, as they are larger than the $100 \mathrm{ps}$ pulse duration of $\mathrm{x}$ rays at the APS and the $120 \mathrm{ps}$ time resolution of the $\mathrm{x}$-ray detector [21] used.

The left panels in Fig. 3 show the measured time response of the interferometer in transmission. The right panels show the energy dependence of the mirror reflectivity and the spectral distribution of the incident $x$-ray photons. The Bragg reflectivity of the mirrors changes with the energy of the incident radiation. Far from the reflectivity maximum the incident radiation pulses traversed the cavity practically without interaction, as in Fig. 3(a). Apart from a few very weak just arising multiple-reflection signals, this plot actually shows the instrument's time resolution function.

The closer the energy of $\mathrm{x}$ rays is to the reflectivity maximum the more significant changes occur. Multiplereflection signals reappearing every 358(1) ps with descending strength are observed. These evenly spaced

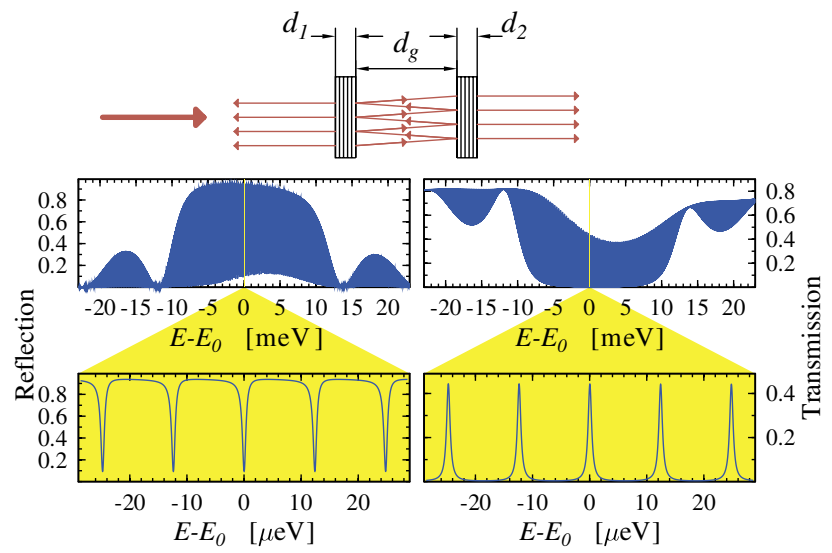

FIG. 2 (color). Top: schematic of an x-ray Fabry-Pérot interferometer with $\alpha-\mathrm{Al}_{2} \mathrm{O}_{3}$ mirrors as in Fig. 1 separated by a gap of $d_{\mathrm{g}}=50 \mathrm{~mm}$. Center and bottom: the appearance of the Fabry-Pérot resonances on the $\mathrm{x}$-ray energy scale in reflection (left), and transmission (right). The central parts on an expanded scale are shown in the bottom graphs. 


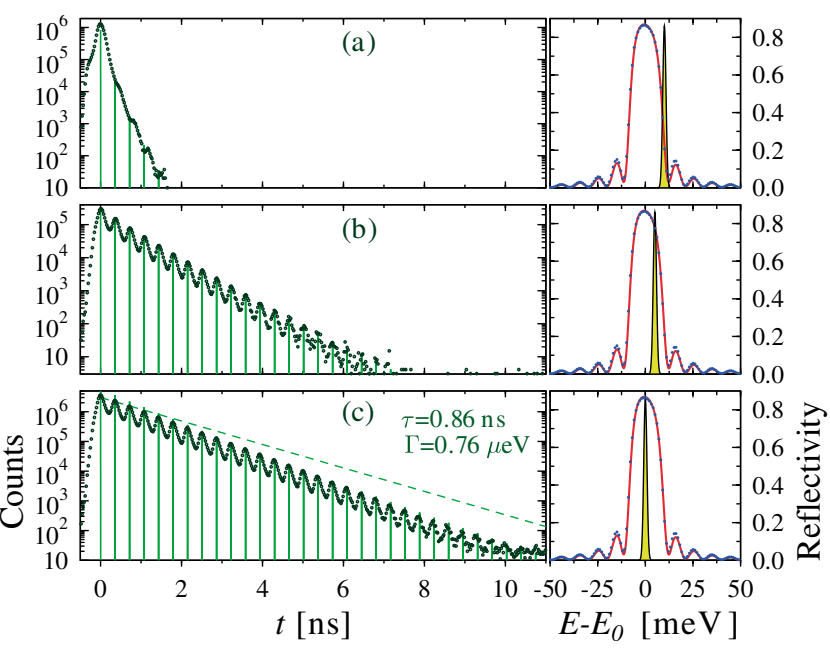

FIG. 3 (color). Left: time response of the interferometer. The sharp green peaks are theoretical fits, without convolution with the time instrumental function. Right: energy dependence of the reflectivity of a single $\alpha-\mathrm{Al}_{2} \mathrm{O}_{3}$ mirror (red lines and dots) and the spectrum of incident $\mathrm{x}$-ray photons (black line) normalized to the peak value of the reflection curve. The center of the spectral distribution $E$ of the incident radiation pulses is detuned (a) $E-E_{0}=10 \mathrm{meV}$ off the peak reflectivity, (b) $E-E_{0}=5 \mathrm{meV}$, and (c) tuned to the peak reflectivity of the mirrors: $E-E_{0}=0$, respectively. The spectral width of the incident radiation pulses is $2 \mathrm{meV}$; pulse duration is 100 ps. The 0.86-ns-decay constant in (c) corresponds to 0.76- $\mu \mathrm{eV}$-broad transmission Fabry-Pérot resonances.

signals appear as a result of $\mathrm{x}$ rays bouncing back and forth between the mirrors. When the $\mathrm{x}$-ray energy coincides with the reflectivity maximum, a train of more than 30 peaks is observed due to $\simeq 60$ reflections; see Fig. 3(c). Such a long train of pulses is observed not only due to a high reflectivity of the mirrors. Also, the reflecting atomic planes of the mirrors should be set parallel. The mirrors could be set parallel with an accuracy of better than $0.35 \mu \mathrm{rad}$ by maximizing the intensity of the most delayed pulses in the train. To do so, the angular position of the second mirror relative to the first one was changed by using a specially designed two-axis nanoradian stage [5]. The time response under these conditions becomes exponential. The longest decay time measured was $\tau \simeq$ $0.86(1) \mathrm{ns}$. Such a decay time corresponds to an energy width of $\Gamma \simeq 0.76(1) \mu \mathrm{eV}$ for the transmission resonances. By using $t_{\mathrm{f}}=358(1) \mathrm{ps}$, one derives for the finesse of the interferometer $F=2 \pi \tau / t_{\mathrm{f}} \simeq 15$, and for the gap between the mirrors $d_{\mathrm{g}}=53.7 \mathrm{~mm}$.

The solid lines in the left panels of Fig. 3, which are a set of very short $(\simeq 2 \mathrm{ps})$ pulses, show the numerically calculated time spectra using the theory of x-ray FabryPérot interferometers $[5,13]$ without any free parameter. The shortness of the pulses is due to the interference of a very large number $\left(\Delta E / E_{\mathrm{f}} \simeq 160\right)$ of Fabry-Pérot resonances simultaneously excited by the $\Delta E=2 \mathrm{meV}$ broad incident radiation spectrum. No convolution with the instrumental function (pulse width and detector resolution) was made. A very good agreement between the experimental and calculated spectra is observed in almost all cases studied so far. To obtain the agreement with the experimental spectrum in Fig. 3(c), a small correction to the energy of the incident photons was necessary. The best fit was achieved by assuming an excitation $3 \mathrm{meV}$ off the reflectivity maximum. The discrepancy is attributed to a drift of the $x$ ray's energy during the two-hour measurement run. The dashed line in the left panel of Fig. 3(c) shows the envelope of the time response with $\tau=1.1 \mathrm{~ns}$ expected for excitation in the maximum at $E-E_{0}=0$. In short preliminary measurements it was measured as $\tau=1.05$ ns.

About $F$ interfering beams are sufficient to obtain an almost perfect interference pattern with sharp FabryPérot resonances $[5,13]$. We observe $\simeq 30$ periods, i.e., twice of this. One has to be, however, sure that these beams interfere. The next experiment demonstrates interference of the beams reflected from the mirrors.

Figure 4 shows the energy dependence of the reflectivity (a), (b), and transmissivity $\left(a^{\prime}\right),\left(b^{\prime}\right)$, of the interferometer. Experimental data are shown by the circles connected with the dotted lines. In the cases of Figs. 4(a) and 4(a'), the atomic planes of the both crystals are adjusted parallel to better than $0.35 \mu \mathrm{rad}$. The period of the thickness

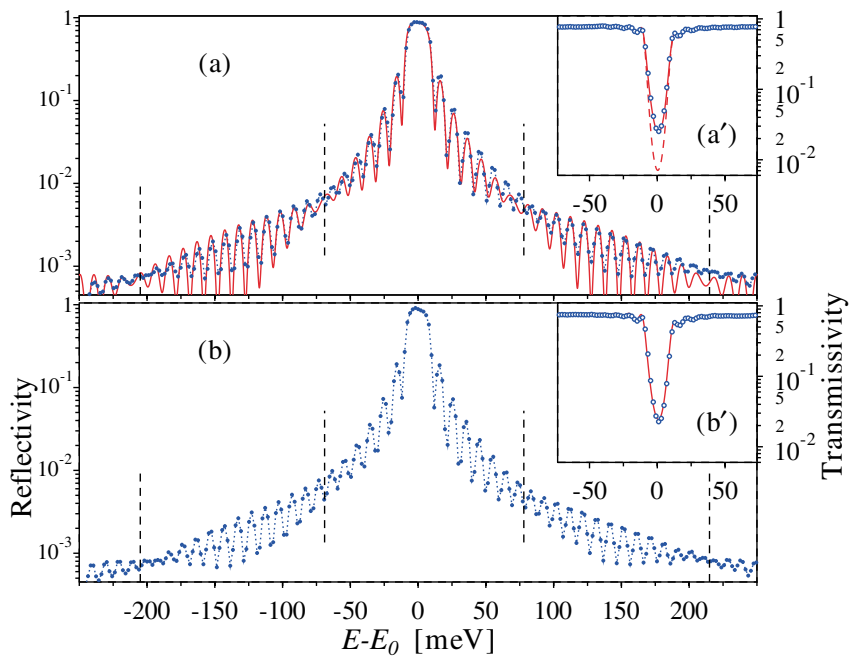

FIG. 4 (color). Energy dependence of the reflectivity (a),(b), and transmissivity $\left(\mathrm{a}^{\prime}\right),\left(\mathrm{b}^{\prime}\right)$, of the two-crystal interferometer. $X$ rays are at normal incidence to the first crystal. (a), $\left(a^{\prime}\right)$ The atomic planes of both crystals are adjusted parallel to better than $0.35 \mu \mathrm{rad}$. The solid red lines are theoretical spectra calculated by the theory of $\mathrm{x}$-ray Fabry-Pérot interferometers $[12,5,13]$ and averaged over the $2 \mathrm{meV}$ bandwidth of the incident radiation. The dashed line in $\left(\mathrm{a}^{\prime}\right)$ is the product of the transmissivities through the mirrors 1 and 2, i.e., describes the transmissivity through a system of two noninteracting mirrors in Bragg backscattering. (b), $\left(b^{\prime}\right)$ Mirror 2 is tilted by $3 \mu \mathrm{rad}$ from the parallel state. The red solid line in $\left(\mathrm{b}^{\prime}\right)$ is the same as in $\left(\mathrm{a}^{\prime}\right)$. Vertical dashed lines indicate the nodes of beatings in (a). 
oscillations fits perfectly to that of the theoretical curve in Fig. 1(a). While the period fits perfectly, the theoretical curve in Fig. 1(a) does not agree with the experimental data in Fig. 4(a) in other respects. Additional periodical modulation - a beat pattern - of the thickness oscillations with four nodal points is observed. Furthermore, the measured reflectivity in the antinodes of the beat pattern is a factor of 2 in excess of the single crystal reflectivity. Already these two features clearly demonstrate that a two-crystal interferometer is realized. The energy separation between the nodal points $\delta E_{\mathrm{d}}=140(3) \mathrm{meV}$ corresponds to a quantity of length $h c / 2 \delta E_{\mathrm{d}}=4.4(2) \mu \mathrm{m}$, which agrees with the difference in the mirror thicknesses $\delta d=d_{1}-d_{2}$ obtained from the fits of the spectra in Figs. 1(a) and 1(b). Therefore, the beat pattern appears due to a slightly different thickness of the crystal mirrors. The observed beating demonstrates unequivocally the interference of the waves reflected from the two mirrors.

The measured spectra in Figs. 4(a) and 4(a $\left.\mathrm{a}^{\prime}\right)$ agree almost perfectly with the spectra evaluated by the theory of x-ray Fabry-Pérot interferometers [12,5,13]. The red solid lines in Figs. 4(a) and $4\left(\mathrm{a}^{\prime}\right)$ present the spectra calculated for monochromatic $\mathrm{x}$ rays (as in Fig. 2) and then averaged over the $2 \mathrm{meV}$ bandwidth of the incident radiation. No free parameters were used. All the details, i.e., the beat pattern in the reflection spectrum, the transmissivity of the system, are described by the theory. The transmissivity is higher than that of two independent mirrors - shown by the red dashed line in Fig. $4\left(\mathrm{a}^{\prime}\right)-$ as it should be for a Fabry-Pérot interferometer.

The beat pattern in Fig. 4(a) can be easily destroyed by tilting the second crystal. Tilting by an angle of $\simeq 3 \mu \mathrm{rad}$ is enough to blur the nodes closest to the reflectivity maximum, as shown in Fig. 4(b). With increasing the tilt, the beat pattern disappears at all, and the reflectivity of the two-crystal system transforms to that of the first mirror, as in Fig. 1(a).

The reported experimental result bears direct evidence of the successful performance of the used two-crystal $\mathrm{x}$-ray interferometer. This brief discussion illustrates that the present design of the x-ray Fabry-Pérot interferometer can be implemented in physical experiments. In this respect, it is important to note, that sapphire is transparent for visible light. This opens up a possibility to realize combined optical-x-ray Fabry-Pérot interferometers, as optical mirrors can be fabricated by thin film metalization of the sapphire x-ray mirrors.

In summary, we have demonstrated an interferometer for hard $\mathrm{x}$ rays with two back-reflecting sapphire crystal mirrors - a prototype x-ray Fabry-Pérot interferometer. A finesse of 15 and $0.76 \mu \mathrm{eV}$ broad Fabry-Pérot transmission resonances is measured by the time response of the interferometer. Interference patterns are observed directly in spectral dependences of reflectivity. These feasibility studies were performed for future research into the realization of (i) combined optical-x-ray FabryPérot interferometers with the purpose of bridging directly the optical and $\mathrm{x}$-ray domains and (ii) interference filters with $\mu \mathrm{eV}$ spectral resolution for studying dynamics of solids, liquids, and macroscopic biological molecules.

Discussions with P. Becker (PTB, Braunschweig) are gratefully acknowledged. We thank A. Alatas, W. Sturhahn, and J. Zhao for assistance with measurements at the APS. Use of HASYLAB (DESY, Hamburg) and APS (ANL, Argonne) facilities is highly appreciated.

*Electronic address: Yuri.Shvydko@desy.de

[1] C. Fabry and A. Pérot, Ann. Chim. Phys. 7, 115 (1899).

[2] M. Born and E. Wolf, Principles of Optics (Pergamon Press, Oxford, New York, 1980).

[3] J. M. Vaughan, The Fabry-Perot Interferometer (Hilger, Bristol, England, Philadelphia, 1989).

[4] A. Steyerl and K.-A. Steinhauser, Z. Phys. B 34, 221 (1979).

[5] Yu. V. Shvyd'ko, Habilitationsschrift, Universität Hamburg, DESY, Hamburg, 2002 (DESY-Thesis-2002028, ISSN 1435-8085, www-library.desy.de/diss02.html).

[6] Currently they are related indirectly via the silicon lattice constant measurements [7-9].

[7] R. D. Deslattes and A. Henins, Phys. Rev. Lett. 31, 972 (1973).

[8] P. Becker, K. Dorenwendt, G. Ebeling, R. Lauer, W. Lucas, R. Probst, H.-J. Radermacher, G. Reim, P. Seyfried, and H. Siegert, Phys. Rev. Lett. 46, 1540 (1981).

[9] G. Basile, A. Bergamin, G. Cavagnero, G. Mana, E. Vittone, and G. Zosi, Phys. Rev. Lett. 72, 3133 (1994).

[10] A. Caticha and S. Caticha-Ellis, Phys. Status Solidi (a) 119, 643 (1990).

[11] A. Caticha, K. Aliberty, and S. Caticha-Ellis, Rev. Sci. Instrum. 67, 3380 (1996).

[12] V. G. Kohn, Yu. V. Shvyd'ko, and E. Gerdau, Phys. Status Solidi (b) 221, 597 (2000).

[13] Yu. V. Shvyd'ko, X-Ray Optics. High Energy Resolution Applications (Springer, Berlin, 2003).

[14] K.-D. Liss, R. Hock, M. Gomm, B. Waibel, A. Magerl, M. Krisch, and R. Tucoulou, Nature (London) 404, 371 (2000).

[15] Yu. V. Shvyd'ko and E. Gerdau, Hyperfine Interact. 123/ 124, 741 (1999).

[16] J. P. Sutter, E. E. Alp, M.Y. Hu, P. L. Lee, H. Sinn, W. Sturhahn, and T.S. Toellner, Phys. Rev. B 63, 094111 (2001)

[17] Yu. V. Shvyd'ko, E. Gerdau, M. Lerche, M. Lucht, H.-C. Wille, E. E. Alp, and P. Becker, Hyperfine Interact. C (to be published).

[18] F. Schmid, C. P. Khattak, and D. M. Felt, Am. Ceram. Soc. Bull. 73, 39 (1994).

[19] W. M. Chen et al., Phys. Status Solidi (a) 186, 365 (2001).

[20] T. S. Toellner, Hyperfine Interact. 125, 3 (2000).

[21] S. Kishimoto, Rev. Sci. Instrum. 63, 824 (1992). 